\title{
COHERENT MULTIPERIOD RISK ADJUSTED VALUES AND BELLMAN'S PRINCIPLE
}

\author{
Philippe Artzner, Université Louis Pasteur \\ Freddy Delbaen, Eidgenössische Technische Hochschule \\ JeAn-Marc Eber, LeXifi \\ David Heath, Carnegie Mellon University \\ HyejIn KU*, York University
}

November 16, 2004

Philippe Artzner, Institut de Recherche Mathématique Avancée, 7 rue René Descartes, F 67084 Strasbourg, France, tel. +33 390240 204, artzner@math.u-strasbg.fr 
Abstract. Starting with a time-0 coherent risk measure defined for "value processes", we also define risk measurement processes. Two other constructions of measurement processes are given in terms of sets of test probabilities. These latter constructions are identical and are related to the former construction when the sets fulfill a stability condition also met in multiperiod treatment of ambiguity as in decision-making. We finally deduce risk measurements for the final value of locked-in positions and repeat a warning concerning Tail-Value-at-Risk.

Keywords. Bellman's principle, capital requirement, coherence, risk-adjusted values, stability by pasting, time consistency. 


\section{INTRODUCTION}

One-period models of risk measurement consider neither the source of possibly required extra capital at the beginning of an holding period nor the actual consequences of a "bad event" at the end of the same period. These points become relevant for multiperiod risk because:

- first, the availability of intermediate information requires taking into account intermediate monitoring by supervisors and/or shareholders of a position even if it is locked-in. Intermediate markets may be missing, but release of information over time does matter at date 0 , for instance because of intermediate audits, see Bennet (2001), or possible actions of competitors,

- second, the possibility of intermediate actions, the availability of extraneous cash-flows and of possible capital in- or outflows require handling sequences of future, unknown, "values",

- third, with one period of uncertainty, capital serves as a buffer at the initial date and as shareholders' wealth at the final date. Intermediate dates, whether deterministic or random, raise the question of the nature of capital (valued in a market or accounting way) at such dates.

This paper is organised as follows.

Section 1 briefly reviews the one-period theory with general probability spaces. We define risk-adjusted values which are more convenient in the multiperiod case than their negative, the traditional risk measures.

Section 2 is the core of the paper. Its first part is devoted to the axiomatics of initial coherent measurement of the risk of "value" processes with our usual emphasis on acceptability. It is intended for supervision of behaviour of "values" on trajectories, a difference with the current literature, see Cvitanić and Karatzas (1999), Föllmer and Schied (2002a, 2002b), Nakano (2003), Roorda, Engwerda and Schumacher (2002), as well as Riedel (2002) (see the end of our Section 4.2).

The second part of Section 2 introduces risk measurement at intermediate stopping times, the acceptance at these times and finally risk measurement processes.

Section 3 prepares a particular construction of multiperiod measurement of risk by "pasting" test probabilities, using the likelihood processes. This approach allows for combining different risk attitudes over time. Sets of test probabilities which are stable by pasting appeared as "rectangular sets" in "multiperiod decision theory with ambiguity" of Epstein and Schneider (2003) and as generalized Bayes rules in Wang (2003). The Epstein-Schneider approach is the multiperiod generalisation of "decision under ambiguity" as studied in Gilboa and Schmeidler (1989). Our definitions work with general state spaces.

Section 4 also constructs two risk-measurement processes of multiperiod-risk by using sets of test probabilities, as in the representation of one-period risk measures. We compare them to each other and to the risk measurement process of the general theory of Section 2. Stability of the set of test probabilities is shown to be equivalent to Bellman's principle and to time consistency as in Wang $(1996,1999)$.

Section 5 deals with multiperiod-measurement of a final values, no matter how they were obtained. They could come e.g. from a locked-in position. Again two constructions are given, using either succesive conditional expectations with respect to all test probabilities or using a recursive procedure. They are identical if and only if the set of test probabilities is stable. We comment on the case of Tail-Valueat-Risk. 


\section{REVIEW OF ONE-PERIOD ACCEPTABILITY}

In the one-period case, the theory of coherent risk measures is best approached, see Artzner et al. (1997, p. 69, 1999a, Section 2.2), Delbaen (2000, 2002), Föllmer and Schied (2002a, 2002b), Heath (1998), by taking the primitive object to be an "acceptance set". It is a set of bounded future random net worths on a probability space $\left(\Omega, \mathcal{F}, \mathbb{P}_{0}\right)$, also simply called "values", which satisfies some axioms of coherence on the part of a regulator or supervisor, either internal or external.

Remark 1. In a one period model of risk measurement, the value at the end of the period may involve an assessment of still future payments like a market value or some form of net worth, e.g. fair value, when markets are missing (as for most insurance liabilities).

Remark 2. The risk measure of a future value is the minimum amount (of numéraire, see below) which has to be added to a current position to make it acceptable. It will be convenient, in the multiperiod case, to work with risk-adjusted values defined as the negative of risk measures: this simplifies the presentation of recursive risk measurement (see for example Bellman's principle in Theorem 4.2, (iii) and Remark 1 following the statement of Theorem 5.1). The risk adjusted measurement of a "value" is then the maximum amount (of numéraire, see below) that can be subtracted from it at the initial date while keeeping it acceptable. The values with positive risk-adjusted measurement are the values that are accepted.

Remark 3. The terminology "risk-adjusted value" is heavy-handed but we want to address the fact that even with pricing under a "risk-neutral" also called "riskadjusted" probability measure, a government bond and a dotcom share of the same current price are very different assets in terms of their future developments.

Remark 4. We express everything via a numéraire, for example the money market account or a default-free zero-coupon long bond - doing so in Artzner et al. (1999a) would have made $r=1$ in Definition 2.2 there. Because of the translation axiom, it is only after this choice of numéraire that risk measures can be qualified as "monetary" (see Föllmer and Schied (2002, 2004)).

The axioms of coherence for acceptance sets or for risk measures (see Artzner et al. (1999a), Sections 2.2 and 2.4, Delbaen (2002), Definition 3, Delbaen (2000), Definition 2.1) translate for coherent risk-adjusted values into:

- monotonicity: for all $X$ and $Y$, if $X \geq Y$ then $\pi(X) \geq \pi(Y)$,

- translation invariance: if $a$ is a constant then for all $X, \pi(a+X)=a+\pi(X)$,

- positive homogeneity: if $\lambda \geq 0$ then for all $X, \pi(\lambda X)=\lambda \pi(X)$,

- superadditivity: for all $X$ and $Y, \pi(X+Y) \geq \pi(X)+\pi(Y)$.

With a general probability space $\left(\Omega, \mathcal{F}, \mathbb{P}_{0}\right)$ a continuity property is in order. The Fatou property for a risk-adjusted value $\pi$ is defined as: for any sequence $\left(X_{n}\right)_{n \geq 1}$ of functions, $\left\|X_{n}\right\|_{\infty} \leq 1$, and converging to $X$ in probability, $\pi(X) \geq \lim \sup \pi\left(X_{n}\right)$, see Delbaen (2002), Definition 4 or Delbaen(2000), Definition 3.1.

The representation result (see Proposition 4.1 in Artzner et al. (1999a) for the case of a finite $\Omega$, Theorem 6 in Delbaen (2002) or Theorem 3.2 in Delbaen (2000) for the infinite $\Omega$ case) states the following:

One-period result. For any coherent risk-adjusted value $\pi: L^{\infty} \rightarrow \mathbb{R}$, having the Fatou property, there exists a convex $L^{1}\left(\mathbb{P}_{0}\right)$-closed set $\mathcal{P}$ of $\mathbb{P}_{0}$-absolutely 
continuous probabilities on $(\Omega, \mathcal{F})$ called generalised scenarios or test probabilities, such that $\pi(X)=\inf _{\mathbb{P} \in \mathcal{P}} \mathbf{E}_{\mathbb{P}}[X]$.

A future value $X$ is accepted at the initial date if and only if for each test probability $\mathbb{P} \in \mathcal{P}$ we have $\mathbf{E}_{\mathbb{P}}[X] \geq 0$.

\section{RISK-MEASUREMENT AT INITIAL AND FOLLOWING DATES}

This section explores one general way of entering the multi-period world: we search first for an initial measurement of multi-preiod risks processes (resulting often from strategies). We then define measurements at future random times, providing thereby risk measurement processes.

We only treat the discrete time case here, see Cheridito, Delbaen and Kupper (2002) for the continuous time case.

\subsection{Multiperiod information.}

Uncertainty over finitely or countably many periods will be described by the probability space $\left(\Omega, \mathcal{F}, \mathbb{P}_{0}\right)$ and by the filtration $\left(\mathcal{F}_{n}\right)_{0 \leq n<\infty}$ such that $\vee_{n} \mathcal{F}_{n}=\mathcal{F}$.

The mathematics of the one-period theory shall be used by looking at (risk) processes as functions on the set of (date-event) pairs which are measurable for the optional sigma-field. The one-period representation result shall provide test probabilities which we shall interpret.

Of course this approach imposes to discuss the interpretation of the axioms of the one-period case. In other words what is being modelled by the processes for which we will define the risk adjusted measurement? The monotonicity and the translation invariance axioms are crucial and we incline to find them better adapted to cumulative cash-flow processes than to cash-flow processes (see for example a different translation invariance property in the paper of Riedel (2002) - in the case of a finite state space - and its related consequence, as we will point out at the end of our Section 4.2).

From the modelling viewpoint we even prefer to work with "value processes" instead of working with cumulative cash-flows. We admit that e.g. changes in asset valuation and provisions, can be passed on as "cash-flows" in some sort of profit and loss accounting, thereby enlarging the interpretation of "cash-flows". In continuous time models value processes are often related to Brownian motion or other Lévy processes having trajectories of infinite variation. Therefore value processes in such models cannot be represented as an indefinite integral of a cash-flow.

Notation. In this paper, particularly Sections 2 and 4 , the domain where we define risk measurement is the set, denoted $\mathcal{G}$, of bounded adapted processes on the filtered space $\left(\Omega,\left(\mathcal{F}_{n}\right)_{0 \leq n<\infty}, \mathbb{P}_{0}\right)$. These processes will be simply called "value" processes.

Many meanings can be given to the word "value" (keeping in mind that for risk supervision purposes we always consider net positions):

- market values of equity,

- accounting values of equity i.e. shareholders' equity or "book value",

- liquidation values,

- in insurance: "shareholders' surplus" as initial capital plus premium received and invested minus claims paid or reserved, see 5.3 in Artzner et al. (1999a), 
We thus deal with concepts which are both prospective (marking to market, reserving) and retrospective (cumulatively "counting the beans" received or given at each date-event). The generality of the meaning of values allows one to use the theory for risk management with risk-measurements by various classes of stakeholders: creditors or regulators, managers, and shareholders, see Artzner et al. (1999b) for such a (one-period) study. This generality also allows to consider individual decision theory in the framework of risk measurement, as was initiated by Heath (1998).

Definition 2.1. (transforming processes into variables) Given a probability space $\left(\Omega, \mathcal{F}, \mathbb{P}_{0}\right)$ and a filtration $\left(\mathcal{F}_{n}\right)_{0 \leq n<\infty}$, the probability space $\left(\Omega^{\prime}, \mathcal{F}^{\prime}, \mathbb{P}_{0}^{\prime}\right)$ is built as follows: $\Omega^{\prime}$ is the product space $\Omega \times \mathbb{N}, \mathcal{F}^{\prime}$ is the $\sigma$-field on $\Omega^{\prime}$ whose elements are unions of families $\left(E_{n} \times\{n\}\right)_{n}, 0 \leq n<\infty$, with $E_{n} \in \mathcal{F}_{n}$ for each $n$. The reference probability $\mathbb{P}_{0}^{\prime}$ is defined on $\left(\Omega^{\prime}, \mathcal{F}^{\prime}\right)$ by the equality:

$$
\mathbb{P}_{0}^{\prime}\left[\bigcup_{0 \leq n<\infty}\left\{E_{n}\right\} \times\{n\}\right]=\sum_{0 \leq n<\infty} \mu_{n} \mathbb{P}_{0}\left[E_{n}\right]
$$

with $\left(\mu_{n}\right)_{n}$ a given sequence where for each $n, \mu_{n}>0$, and $\sum_{0 \leq n<\infty} \mu_{n}=1$.

The set $\mathcal{G}$ of bounded adapted processes, becomes the set of bounded measurable functions on the measurable space $\left(\Omega^{\prime}, \mathcal{F}^{\prime}\right)$. On $\mathcal{G}$, we use the topology coming from the duality between $L^{1}\left(\Omega^{\prime}, \mathcal{F}^{\prime}, \mathbb{P}_{0}^{\prime}\right)$ and $L^{\infty}\left(\Omega^{\prime}, \mathcal{F}^{\prime}, \mathbb{P}_{0}^{\prime}\right)$. A set is closed in $\mathcal{G}$ if and only if it is closed with respect to the weak* topology coming from $L^{1}\left(\Omega^{\prime}, \mathcal{F}^{\prime}, \mathbb{P}_{0}^{\prime}\right)$. The case of unbounded value processes is the topic of on-going research.

\subsection{The cone of value processes acceptable at the initial date.}

A supervisor, risk manager or regulator will, as in the one-period case, decide at date 0 upon a subset of $\mathcal{G}$ denoted $\mathcal{A}_{c c}$, of acceptable value processes and this leads to risk measurement as follows.

Definition 2.2. (coherent acceptance set) A coherent acceptance set of value processes is a closed convex cone of $\mathcal{G}$, with vertex at the origin, containing the positive orthant and intersecting the negative orthant only at the origin.

Definition 2.3. (coherent risk adjusted value) The risk adjusted value associated with the coherent cone $\mathcal{A}_{c c}$ is defined as $\pi(X)=\sup \left\{m \mid X-m \in \mathcal{A}_{c c}\right\}$.

As in the one-period case changing numéraire is not a problem for the acceptance set but becomes a problem for the derived risk measurement (see Artzner et al. (1999b), Remark 2.5 and Artzner, Delbaen and Koch (2005)).

The coherence assumption on $\mathcal{A}_{c c}$ ensures that the associated risk adjusted valuation is "coherent", i.e. it satisfies the axioms listed at the end of Section 1.

As in Delbaen $(2000,2002)$ we have the following link between the Fatou property and the closedness of acceptance sets:

Proposition 2.1. For a convex cone $\mathcal{A}_{c c} \subset \mathcal{G}$, containing the cone of nonnegative processes, the following statements are equivalent

(1) $\mathcal{A}_{c c}$ is $\sigma\left(\mathcal{G}, L^{1}\left(\mathbb{P}_{0}^{\prime}\right)\right)$ closed

(2) the associated measurement $\pi$ satisfies the Fatou property: if $X^{k} \in \mathcal{G}$ is a sequence uniformly bounded by 1 and such that $X^{k} \rightarrow X$ in $\mathbb{P}_{0}^{\prime}$ probability, then $\pi(X) \geq \varlimsup \pi\left(X^{k}\right)$ 
(3) the associated measurement $\pi$ satisfies the property: if $X^{k}$ is a sequence in $\mathcal{G},\left\|X_{n}^{k}\right\|_{\infty} \leq 1$ and such that for all $n, X_{n}^{k} \rightarrow X_{n}$ in probability, then $\pi(X) \geq \overline{\lim } \pi\left(X^{k}\right)$.

\subsection{Translation of the one-period representation result.}

Thanks to the identification between value processes and random variables on $\left(\Omega^{\prime}, \mathcal{F}^{\prime}\right)$ we directly deduce from the study of the one-period case (see Section 1) that, given a coherent risk adjusted value measurement $\pi^{0}$ with the Fatou property, there is a closed convex set $\mathcal{P}^{\prime}$ of probabilities on $\left(\Omega^{\prime}, \mathcal{F}^{\prime}\right)$, absolutely continuous w.r.t. $\mathbb{P}_{0}^{\prime}$, such that:

$$
\text { for each } X \in \mathcal{G}, \pi^{0}(X)=\inf _{\mathbb{Q}^{\prime} \in \mathcal{P}^{\prime}} \mathbf{E}_{\mathbb{Q}^{\prime}}[X]
$$

Remark. One could consider more general acceptance sets than convex cones, as was done in Föllmer and Schied $(2002,2004)$ (see also their use in Heath (1998), to represent the risk measurement affine constraints imposed by the shareholders of a firm).

Each "test probability" $\mathbb{Q}^{\prime} \in \mathcal{P}^{\prime}$ above can be described by its density $f^{\prime}$ with respect to $\mathbb{P}_{0}^{\prime}$. This density must first of all be a random variable on $\left(\Omega^{\prime}, \mathcal{F}^{\prime}\right)$ which we can represent as $f^{\prime}=\left(f_{n}\right)_{0 \leq n<\infty}$ where each $f_{n}$ is a non-negative and $\mathcal{F}_{n}$-measurable function on $\Omega$ such that $\sum_{0 \leq n<\infty} \mu_{n} \mathbf{E}_{\mathbb{P}_{0}}\left[f_{n}\right]=1$. We then have for each $X=\left(X_{n}\right)_{0 \leq n<\infty}, \mathbf{E}_{\mathbb{Q}^{\prime}}[X]=\sum_{0 \leq n<\infty} \mu_{n} \mathbf{E}_{\mathbb{P}_{0}}\left[f_{n} X_{n}\right]$. Defining the increasing adapted process $A$ by $A_{n}=A_{n-1}+\mu_{n} f_{n}$, with $A_{-1}=0$, we get that $\mathbf{E}_{\mathbb{P}_{0}}\left[A_{\infty}\right]=1$ and we obtain the representation result by minimizing over the set $\mathcal{A}$ of processes corresponding to the set of test probabilities $\mathbb{P}^{\prime} \in \mathcal{P}^{\prime}$ :

Proposition 2.2. For each "initial date" coherent risk-adjusted valuation $\pi^{0}$ of value processes with the Fatou property, there is a closed convex set $\mathcal{A}$ of positive adapted non-decreasing processes $A$ with $\mathbf{E}_{\mathbb{P}_{0}}\left[A_{\infty}\right]=1$ such that for each value process $X$ :

$$
\pi^{0}(X)=\inf _{A \in \mathcal{A}} \mathbf{E}_{\mathbb{P}_{0}}\left[\sum_{0 \leq n<\infty} X_{n}\left(A_{n}-A_{n-1}\right)\right]
$$

Remark 1. The set $\mathcal{A}$ can be taken to be a closed set in the normed space of processes with integrable variation, where $\|A\|=\mathbf{E}_{\mathbb{P}_{0}}\left[\left|A_{0}\right|+\sum_{0 \leq n<\infty}\left|A_{n+1}-A_{n}\right|\right]$.

Remark 2. If, with $N$ periods, the value process $X$ is a $\mathbb{P}_{0}-$ martingale, the general expression for $\pi^{0}(X)$ simplifies to $\inf _{A \in \mathcal{A}} \mathbf{E}_{\mathbb{P}_{0}}\left[X_{N} A_{N}\right]$. In words, for our risk measurement method, "only the final value matters" when dealing with $\mathbb{P}_{0}$-martingales. Being defined on the whole of $\mathcal{G}$, our method handles more general business conditions than "marking to (complete) markets".

Remark 3. The increments $\left(A_{n}-A_{n-1}\right)$ are a way to describe how the evolution of the values is supervised and taken into account. As the reader can see the choice of the initial sequence $\left(\mu_{n}\right)_{n}$, in Definition 2.1, is irrelevant.

Remark 4. Works like Cvitanić and Karatzas (1999), Föllmer and Schied (2002a, 2002b), Nakano (2003), also have several periods for portfolio construction, and measure the risk of final wealths only. The risk measures considered do not take 
into account what happens on trajectories at intermediate dates. Remark 2 above explains a contrario the difference and the first two examples below will illustrate. The paper by Jaschke and Küchler (2001) mentions processes as potential objects of risk measurement (at the intial date only) but does not present either the switch from $\Omega$ to $\Omega^{\prime}$ (this Section) nor the use of the representing test probabilities (our Section 4).

\subsection{Examples of initial measurement of multiperiod risk.}

Example 2.1.

The measurement $\pi^{0}$ defined for each value process $X$ by $\pi^{0}(X)=\mathbf{E}_{\mathbb{P}_{0}}\left[X_{\sigma}\right], \sigma$ a stopping time, is a coherent risk-adjusted measurement, describing "how good or bad processes are at date $\sigma$ ".

Define, if $\mathbb{P}_{0}[\sigma<\infty]>0$, the non-decreasing process $A^{\sigma}$ by $A_{n}^{\sigma}=\frac{1}{\mathbb{P}_{0}[\sigma<\infty]} \mathbf{1}_{\{\sigma \leq n\}}$. Then for each $X, \pi^{0}(X)=\mathbf{E}_{\mathbb{P}_{0}}\left[\sum_{0 \leq n<\infty} X_{n}\left(A_{n}^{\sigma}-A_{n-1}^{\sigma}\right)\right]$.

Example 2.2.

The measurement $\pi^{0}$ defined by $\pi^{0}(X)=\mathbf{E}_{\mathbb{P}_{0}}\left[\inf _{0 \leq n \leq N} X_{n}\right]$ is a coherent riskadjusted measurement related to the random times $\bar{\tau}_{X}$ where

$$
\left.\bar{\tau}_{X}(\omega)=\operatorname{argmin}_{n: n \leq N}\left(X_{n}(\omega)\right)\right) .
$$

Since dates of extrema are not necessarily stopping times, it is a challenge to represent $\pi^{0}$ with adapted processes! To do this we define for any random time $\tau$ the process $A^{\tau}$ where $A_{n}^{\tau}=A_{n-1}^{\tau}+\mathbf{E}_{\mathbb{P}_{0}}\left[\mathbf{1}_{C_{n}} \mid \mathcal{F}_{n}\right]$ with $C_{n}=\{\tau=n\}$. Denote by $\mathcal{A}$ the closed convex hull of all such $A^{\tau}$. For each process $X$ we have $\mathbf{E}_{\mathbb{P}_{0}}\left[\sum_{0 \leq n \leq N} X_{n} \mathbf{1}_{C_{n}}\right]=\mathbf{E}_{\mathbb{P}_{0}}\left[\sum_{0 \leq n \leq N} X_{n}\left(A_{n}^{\tau}-A_{n-1}^{\tau}\right)\right]$. Using the random times $\bar{\tau}_{X}$ defined above we find that $\pi^{0}$ is given by

$$
\pi^{0}(X)=\inf _{A^{\tau} \in \mathcal{A}} \mathbf{E}_{\mathbb{P}_{0}}\left[\sum_{0 \leq n \leq N} X_{n}\left(A_{n}^{\tau}-A_{n-1}^{\tau}\right)\right]
$$

Example 2.3.

The measurement $\pi^{0}$ defined by $\pi^{0}(X)=\frac{1}{N+1} \mathbf{E}_{\mathbb{P}_{0}}\left[\sum_{0 \leq n \leq N} X_{n}\right]$ is coherent but it would not fulfill the "translation invariance" property of Riedel (2002).

\subsection{Extension of an initial risk measurement to future dates.}

We reach the second part of our program: getting risk-adjusted measurements at future (stopping) times. To keep the presentation simple we suppose that the time interval is finite and we also suppose that the risk adjusted measurements satisfy the Fatou property.

Proposition 2.3. For each coherent risk adjusted measurement $\pi^{0}$, each stopping time $\tau$, and each value process $X$ we have:

(1) the set of all $\mathcal{F}_{\tau}-$ measurable random variables

$\left\{f \mid f\right.$ is $\mathcal{F}_{\tau}-$ measurable and for all $\left.A \in \mathcal{F}_{\tau}: \pi^{0}\left(\mathbf{1}_{A} \mathbf{1}_{\llbracket \tau, \infty \llbracket}(X-f)\right) \geq 0\right\}$, is closed under taking the maximum of a finite number of elements and it has a maximal element, denoted by $\pi_{\tau}^{0}(X)$ and called the risk-adjusted value of the process $X$ at the date $\tau$,

(2) $\pi_{\tau}^{0}$ satisfies

$$
\pi_{\tau}^{0}(X)=\sum_{0 \leq t \leq N} \pi_{t}^{0}(X) \mathbf{1}_{\{\tau=t\}} .
$$


Proposition 2.4. For each stopping time $\tau$ we have:

(1) the mapping $\pi_{\tau}^{0}$ is positive, super-additive, positively homogeneous,

(2) for each $g$ bounded and $\mathcal{F}_{\tau}$-measurable we have

$$
\pi_{\tau}^{0}\left(X+g \mathbf{1}_{\llbracket \tau, N \rrbracket}\right)=\pi_{\tau}^{0}(X)+g,
$$

(3) the mapping $\pi_{\tau}^{0}$ is Fatou. More precisely: for each sequence of processes $X^{n}$ such that $\left\|X^{n}\right\| \leq 1$ and for all $1 \leq t \leq N, X_{t}^{n} \rightarrow X_{t}$ (a.s.), we have $\pi_{\tau}^{0}(X) \geq \lim \sup \pi_{\tau}^{0}\left(X^{n}\right)$, where the limsup is taken in probability.

The proofs of these propositions are left to the reader.

Remark. It is worth emphasizing that the filtration $\left(\mathcal{F}_{n}\right)_{0 \leq n \leq N}$ is a primitive and that for each $X \in \mathcal{G}$ and each $n$, the $\pi_{n}^{0}(X)$ depends on the filtration.

\section{PASTING OF TEST PROBABILITIES}

In contrast with Section 2, we shall keep the test probabilities on the base state space $\Omega$ and shall use extensively conditional expectations for "updating" the measurement of risk as time evolves.

We thank J. Hugonnier for pointing out the work of Epstein and Schneider (2003) on the occasion of the presentation of these ideas in the talk Artzner et al. (2001). There is indeed a touch of utility theory in our work since the cone of values acceptable to the supervisor and the translation invariance define a, special, preference order (see also Heath (1998)). We also thank L. Epstein for a discussion on this topic. See also the paper Wang (2003) and the brief discussion of it in Section 3.2 of Epstein and Schneider (2003).

Nevertheless several points differentiate our Sections 3 and 4, since in Epstein and Schneider (2003):

- preferences are on lottery-valued processes,

- preferences are not required to be translation-invariant,

- preferences are not required to be monotonic,

- the state space is finite.

Notation. For the remainder of the paper we consider only $N$ periods of uncertainty (see Delbaen (2001) for a continuous time approach) and work with a closed convex set $\mathcal{P}$ of "test" probabilities on $\left(\Omega, \mathcal{F}_{N}\right)$ absolutely continuous with respect to $\mathbb{P}_{0}$. For convenience we can assume that the reference probability $\mathbb{P}_{0}$ belongs to the chosen set $\mathcal{P}$.

This section prepares for two specific multiperiod-measurements of risk. We shall combine test probabilities on $\Omega$ by identifying them to their densities with respect to the reference probability $\mathbb{P}_{0}$ and use the likelihood ratio processes.

To avoid problems with probabilities $\mathbb{Q} \in \mathcal{P}$ which are only absolutely continuous with respect to $\mathbb{P}_{0}$ but not equivalent to it, we often use the convex dense set $\mathcal{P}^{e}$ of elements of $\mathcal{P}$ which are equivalent to $\mathbb{P}_{0}$.

\subsection{Stability of the set of test probabilities.}

Any closed convex set $\mathcal{P}$ of test probabilities on $\left(\Omega, \mathcal{F}_{N}\right)$ absolutely continuous with respect to $\mathbb{P}_{0}$ is identified with the closed convex set $\mathcal{Z}_{\mathcal{P}}$ of all the $\mathbb{P}_{0^{-}}$ martingales $Z_{n}^{\mathbb{Q}}=\mathbf{E}_{\mathbb{P}_{0}}\left[\frac{d \mathbb{Q}}{d \mathbb{P}_{0}} \mid \mathcal{F}_{n}\right], 0 \leq n \leq N, \mathbb{Q} \in \mathcal{P}$, see Chow, Robbins and Siegmund (1972), Section 2.1, Dothan (1990), Sections 5.4, 6.4. 
Definition 3.1. (pasting, stability) We say that the set $\mathcal{P}$ of test probabilities is stable if for elements $\mathbb{Q}^{0}, \mathbb{Q} \in \mathcal{P}^{e}$ with associated martingales $Z_{n}^{0}, Z_{n}$, and for each stopping time $\tau$ the martingale $L$ defined as $L_{n}=Z_{n}^{0}$ for $n \leq \tau$ and $L_{n}=Z_{\tau}^{0} \frac{Z_{n}}{Z_{\tau}}$ for $n \geq \tau$ defines an element of $\mathcal{P}$ called the result of pasting $\mathbb{Q}_{0}$ and $\mathbb{Q}$.

The following lemma rephrases the definition of stability:

Lemma 3.1. The stability of the set $\mathcal{P}$ implies that for any three stopping times $\tau \leq \sigma \leq \nu$, the following two sets are equal:

$$
\left\{\left(\frac{Z_{\nu}}{Z_{\sigma}}, \frac{Z_{\sigma}}{Z_{\tau}}\right) \mid Z \in \mathcal{P}^{e}\right\}=\left\{\left(\frac{Z_{\nu}^{\prime}}{Z_{\sigma}^{\prime}}, \frac{Z_{\sigma}}{Z_{\tau}}\right) \mid Z \in \mathcal{P}^{e}, Z^{\prime} \in \mathcal{P}^{e}\right\}
$$

\subsection{An example of a stable set of test probabilities.}

Let the information structure be given by a binomial tree and a random walk $W_{n}=U_{1}+\ldots+U_{n}, 0 \leq n \leq N$, the $\left(U_{n}\right)_{0 \leq n \leq N}$ being \pm 1 valued independent variables with symmetric distribution. If $\mathbb{P}_{0}^{-}$denotes the resulting measure on $\left(\Omega, \mathcal{F}_{N}\right)$, any probability $\mathbb{Q}$ on $\left(\Omega, \mathcal{F}_{N}\right)$ has its density with respect to $\mathbb{P}_{0}$ written as $Z=\prod_{0 \leq n \leq N}\left(1+q_{n} U_{n}\right)$ with the $\left(q_{n}\right)_{0 \leq n \leq N}$ being a predictable process with $\left|q_{n}\right| \leq 1$.

It can then be shown that stability of a closed convex set $\mathcal{P}$ of test probabilities is equivalent to representability of the density processes of elements of $\mathcal{P}$, meaning that for each $n, 0 \leq n \leq N$, there exists a closed convex set $\mathcal{Q}_{n}$ of $[-1,+1]$, described in an $\mathcal{F}_{n-1}$-measurable way, such that the density $Z=\prod_{0 \leq n \leq N}\left(1+q_{n} U_{n}\right)$ is an element of $\mathcal{Z}_{\mathcal{P}}$ if and only if each $q_{n}$ belongs to $\mathcal{Q}_{n}$. The continuous time case is treated in Delbaen (2001) with stochastic exponentials.

A special case of the example is amenable to simple spreadsheet computations. Take for some $d, 0 \leq d \leq 1$, all the sets $\mathcal{Q}_{n}$ to be the interval $[-d,+d]$. The set $\mathcal{P}$ is then the set of all probabilities $\mathbb{Q}$ such that $\frac{d \mathbb{Q}}{d \mathbb{P}_{0}}=Z_{N}$ satisfies $Z_{n}=\mathbf{E}_{\mathbb{P}_{0}}\left[Z_{N} \mid \mathcal{F}_{n}\right]=$ $\left(1+q_{1} U_{1}\right) \ldots\left(1+q_{n} U_{n}\right)$ where $q$ is a predictable process with $|q| \leq d \leq 1$. This is indeed a stable set. As the reader can check we get that $\Psi_{0}(W)=-d N$ which shows in particular the role of the total time required to get complete information. More on this is to be found in Artzner (2002b).

\section{TWO SPECIFIC MULTIPERIOD-MEASUREMENTS OF MULTIPERIOD-RISK}

In contrast with the axiomatic approach of Section 2, we build directly processes of risk-adjusted values by using a set $\mathcal{P}$ of test probabilities on $\left(\Omega, \mathcal{F}_{N}\right)$. We present two constructions and show that they are identical if and only if $\mathcal{P}$ is stable.

\subsection{A risk-adjusted value process seen as a generalized Snell envelope.}

Given test probabilities on $\Omega$, a value process may well be a martingale under some of these but risk-adjusted value processes should only be required to be submartingales under each test probability: since information has been provided, uncertainty of the remaining part decreases and risk-adjusted value increases "on (conditional) average".

Since there is no reason to over-penalize a value process, we want to risk-adjust it by the largest possible process. The theory of the Snell envelope provides the convenient framework (see Chow, Robbins and Siegmund (1972), Section 1.6 and Theorem 4.7, Neveu (1972), Propositions VI.1.1 and VI-1-2, for this as well and for a precise treatment of essential infimum). 
Theorem 4.1. Given the set $\mathcal{P}$ of test probabilities there is for each value process $X \in \mathcal{G}$ a unique process $\bar{\Psi}(X)$ which is the largest of all processes $Y \in \mathcal{G}$ with the following two properties:

(i) $Y \leq X$ as processes,

(ii) for each test probability $\mathbb{Q} \in \mathcal{P}, Y$ is a $\mathbb{Q}$-submartingale.

It is given by the formula

$$
\bar{\Psi}_{N}(X)=X_{N} ; \text { for } 0 \leq n<N, \bar{\Psi}_{n}(X)=X_{n} \wedge \text { ess. } \inf _{\mathbb{Q} \in \mathcal{P} e} \mathbf{E}_{\mathbb{Q}}\left[\bar{\Psi}_{n+1}(X) \mid \mathcal{F}_{n}\right]
$$

Proof. It is clear that $\bar{\Psi}(X)$ satisfies (i) and (ii); for any other process $Y$ with (i) and (ii) satisfied we have as soon as $Y_{n+1} \leq \bar{\Psi}_{n+1}(X)$, for each $\mathbb{Q} \in \mathcal{P}, Y_{n} \leq$ $\mathbf{E}_{\mathbb{Q}}\left[Y_{n+1} \mid \mathcal{F}_{n}\right] \leq \mathbf{E}_{\mathbb{Q}}\left[\bar{\Psi}_{n+1}(X) \mid \mathcal{F}_{n}\right]$. These inequalities being valid for each $\mathbb{Q}$ prove, together with $Y_{n} \leq X_{n}$, that $Y_{n} \leq \bar{\Psi}_{n}(X)$.

\subsection{Stability of test probabilities and Bellman's principle.}

We leave the proof of the following lemma to the reader:

Lemma 4.1. For a fixed stopping time $\sigma$ and for a stable set $\mathcal{P}$, the set

$$
\left\{\mathbf{E}_{\mathbb{Q}}\left[X_{\tau} \mid \mathcal{F}_{\sigma}\right] \mid \tau \geq \sigma \text { is a stopping time and } \mathbb{Q} \in \mathcal{P}^{e}\right\}
$$

is closed for taking minima (also maxima).

Corollary. If $\mathcal{P}$ is stable we also have for every stopping time $0 \leq \sigma$ and every probability measure $\mu \ll \mathbb{P}_{0}$ (not necessarily in $\mathcal{P}$ ) that

$$
\begin{aligned}
\mathbf{E}_{\mu}\left[\text { ess. } \inf _{\mathbb{Q} \in \mathcal{P} e} \mathbf{E}_{\mathbb{Q}}\left[X_{\tau} \mid \mathcal{F}_{\sigma}\right]\right] \\
\quad=\inf \left\{\mathbf{E}_{\mu}\left[\mathbf{E}_{\mathbb{Q}}\left[X_{\tau} \mid \mathcal{F}_{\sigma}\right]\right] \mid \mathbb{Q} \in \mathcal{P}^{e} ; \sigma \leq \tau \text { stopping time }\right\} .
\end{aligned}
$$

The corollary follows from Lemma 4.1 by using Proposition VI-1-1 of Neveu (1972).

Theorem 4.2. Given the set $\mathcal{P}$ of test probabilities, for each value process $X \in \mathcal{G}$, the family $\left(\Psi_{\sigma}(X)\right), \sigma$ a stopping time, where:

$$
\Psi_{\sigma}(X)=\text { ess. } \inf \left\{\mathbf{E}_{\mathbb{Q}}\left[X_{\tau} \mid \mathcal{F}_{\sigma}\right] \mid \tau \geq \sigma \text { is a stopping time, } \mathbb{Q} \in \mathcal{P}^{e}\right\} .
$$

defines a process $\Psi(X)$.

The stability of $\mathcal{P}$ is equivalent to each of the following

(i) for each $\mathbb{Q} \in \mathcal{P}$ and each value process $X \in \mathcal{G}, \Psi(X)$ is a $\mathbb{Q}$-submartingale,

(ii) the mappings $\Psi$ and $\bar{\Psi}$ are equal,

(iii) "Bellman's principle": for each $X \in \mathcal{G}$ and stopping times $\sigma, \tau, \sigma \leq \tau$, we have $\Psi_{\sigma}(X)=\Psi_{\sigma}\left(X^{\tau-}+\Psi_{\tau}\left({ }^{\tau} X\right) \mathbf{1}_{\llbracket \tau, N \rrbracket}\right)$.

Remark. We recall the definitions of $X^{\tau-}$ and ${ }^{\tau} X$ as ${ }^{\tau} X_{n}=0$ for $n<\tau,{ }^{\tau} X_{n}=$ $X_{n}-X_{\tau-1}$ for $n \geq \tau, X_{n}^{\tau-}=X_{n}$ for $n<\tau, X_{n}^{\tau-}=X_{\tau-1}$ for $n \geq \tau$. We remark that ${ }^{\tau}\left({ }^{\tau} X\right)={ }^{\tau} X,\left({ }^{\tau} X\right){ }^{\tau-}=0$ and that $\Psi_{\tau}(X)=\Psi_{\tau}\left({ }^{\tau} X\right)+X_{\tau-1}$.

Proof.

(1) We first show that $\Psi \geq \bar{\Psi}$, which will ensure that if $\Psi(X)$ is - for each $X$ and each $\mathbb{Q} \in \mathcal{P}$ - a $\mathbb{Q}$-submartingale, $\Psi(X)=\bar{\Psi}(X)$ (use Theorem 4.1, 
i.e. if (i) is satisfied so is (ii)). On the other hand (i) follows from (ii) by construction of $\bar{\Psi}$. Since for each $\mathbb{Q} \in \mathcal{P}, \bar{\Psi}(X)$ is a $\mathbb{Q}$-submartingale, for each stopping time $\tau, \bar{\Psi}_{\sigma}(X) \leq \mathbf{E}_{\mathbb{Q}}\left[\bar{\Psi}_{\tau}(X) \mid \mathcal{F}_{\sigma}\right] \leq \mathbf{E}_{\mathbb{Q}}\left[X_{\tau} \mid \mathcal{F}_{\sigma}\right]$. Taking ess. inf over $\mathbb{Q}$ and $\tau$ provides the stated inequality.

(2) We show now that (i) implies stability of $\mathcal{P}$. We suppose that $Z^{1}$ and $Z^{2}$ are two elements in $\mathcal{Z}_{\mathcal{P} e}$ - coming from the measures $\mathbb{Q}^{1}, \mathbb{Q}^{2}-$ and $\sigma$ is a stopping time. Also suppose that the element $Z_{\sigma}^{1} \frac{Z_{N}^{2}}{Z_{\sigma}^{2}}$ is not in the closed convex set $\mathcal{P}$. By the separation theorem for convex sets there is a random variable $f \in L^{\infty}$, so that

$$
\mathbf{E}_{\mathbb{P}}\left[Z_{\sigma}^{1} \frac{Z_{N}^{2}}{Z_{\sigma}^{2}} f\right]<\inf _{\mathbb{Q} \in \mathcal{P}} \mathbf{E}_{\mathbb{Q}}[f]
$$

Defining the process $X$ by $X_{N}=f, X_{n}=\|f\|$ for $n<N$, we find that $\Psi_{0}(X)=\inf _{\mathbb{Q} \in \mathcal{P}} \mathbf{E}_{\mathbb{Q}}[f]$. The left hand side of the displayed inequality can be written as $\mathbf{E}_{\mathbb{Q}^{1}}\left[\mathbf{E}_{\mathbb{Q}^{2}}\left[f \mid \mathcal{F}_{\sigma}\right]\right]$.

This is clearly at least equal to $\mathbf{E}_{\mathbb{Q}^{1}}\left[\Phi_{\sigma}(X)\right]$, a quantity which by the $\mathbb{Q}^{1}$-submartingale property is at least equal to $\Psi_{0}(X)$. This is a contradiction since the right hand side of the displayed inequality is precisely $\Psi_{0}(X)$.

(3) Let us prove that stability implies Bellman's principle (iii). To avoid complicated notation, the measures $\mathbb{Q}, \mathbb{Q}^{\prime}, \mathbb{Q}^{\prime \prime}$ are taken in $\mathcal{P}^{e}$. By definition

$$
\begin{aligned}
& \Psi_{\sigma}(X) \\
& =\text { ess. } \inf _{\mathbb{Q}, \nu \geq \sigma} \mathbf{E}_{\mathbb{Q}}\left[X_{\nu} \mid \mathcal{F}_{\sigma}\right] \\
& =\text { ess. } \inf _{\mathbb{Q}, \nu \geq \sigma} \mathbf{E}_{\mathbb{Q}}\left[\mathbf{E}_{\mathbb{Q}}\left[X_{\nu} \mid \mathcal{F}_{\tau}\right] \mid \mathcal{F}_{\sigma}\right] \\
& =\text { ess. } \inf _{\mathbb{Q}, \nu \geq \sigma} \mathbf{E}_{\mathbb{Q}}\left[X_{\nu} \mathbf{1}_{\nu<\tau}+\mathbf{E}_{\mathbb{Q}}\left[X_{\nu} \mathbf{1}_{\nu \geq \tau} \mid \mathcal{F}_{\tau}\right] \mid \mathcal{F}_{\sigma}\right] \\
& =\text { ess. } \inf _{\mathbb{Q}, \nu \geq \sigma} \mathbf{E}_{\mathbb{Q}}\left[X_{\nu} \mathbf{1}_{\nu<\tau}+\mathbf{1}_{\nu \geq \tau}\left(X_{\tau-}+\mathbf{E}_{\mathbb{Q}}\left[X_{\nu}-X_{\tau-} \mid \mathcal{F}_{\tau}\right]\right) \mid \mathcal{F}_{\sigma}\right]
\end{aligned}
$$

The Corollary of Lemma 4.1 together with Lemma 3.1 allow us to rewrite the result of the simple ess.inf as a compounded expression:

$$
\begin{aligned}
& \Psi_{\sigma}(X) \\
& =\text { ess. } \inf _{\mathbb{Q}^{\prime}, \nu \geq \sigma} \mathbf{E}_{\mathbb{Q}^{\prime}}\left[X_{\nu} \mathbf{1}_{\nu<\tau}\right. \\
& \left.\quad+\mathbf{1}_{\nu \geq \tau} \text { ess. } \inf _{\mathbb{Q}^{\prime \prime}, \nu^{\prime} \geq \tau}\left(X_{\tau-}+\mathbf{E}_{\mathbb{Q}^{\prime \prime}}\left[X_{\nu^{\prime}}-X_{\tau-} \mid \mathcal{F}_{\tau}\right]\right) \mid \mathcal{F}_{\sigma}\right] \\
& =\text { ess. } \inf _{\mathbb{Q}^{\prime}, \nu \geq \sigma} \mathbf{E}_{\mathbb{Q}^{\prime}}\left[X_{\nu} \mathbf{1}_{\nu<\tau}+\mathbf{1}_{\nu \geq \tau}\left(X_{\tau-}+\Psi_{\tau}\left({ }^{\tau} X\right) \mid \mathcal{F}_{\sigma}\right]\right. \\
& =\text { ess. } \inf _{\mathbb{Q}^{\prime}, \nu \geq \sigma} \mathbf{E}_{\mathbb{Q}^{\prime}}\left[\left(X^{\tau-}+\Psi_{\tau}\left({ }^{\tau} X\right)\right)_{\nu} \mid \mathcal{F}_{\sigma}\right] \\
& =\Psi_{\sigma}\left(X^{\tau-}+\Psi_{\tau}\left({ }^{\tau} X\right) \mathbf{1}_{\llbracket \tau, N \rrbracket}\right)
\end{aligned}
$$

(4) Bellman's principle (iii) ensures that

$$
\begin{aligned}
\Psi_{\sigma}(X) & \leq \Psi_{\sigma}\left(\|X\|+\Psi_{\tau}\left({ }^{\tau} X\right) \mathbf{1}_{\llbracket \tau, N \rrbracket}\right) \\
& \leq \text { ess. } \inf \left\{\mathbf{E}_{\mathbb{Q}}\left[\Psi_{\tau}\left({ }^{\tau} X\right) \mid \mathcal{F}_{\sigma}\right] \mid \mathbb{Q} \in \mathcal{P}^{e}\right\}
\end{aligned}
$$

hence for each $\mathbb{Q} \in \mathcal{P}, \Psi_{\sigma}(X) \leq \mathbf{E}_{\mathbb{Q}}\left[\Psi_{\tau}(X) \mid \mathcal{F}_{\sigma}\right]$, so (i) is a consequence of (iii) and the proof of Theorem 4.2 is complete. 
Remark 1. The risk measurement of Example 2.3, Section 2, satisfies Bellman's principle but it is not of the form $\Psi_{0}$ or $\bar{\Psi}_{0}$ for any set $\mathcal{P}$ of test probabilities.

Remark 2. With our sign convention the risk-adjusted measurement of cash-flow streams in Riedel (2002) would appear as

$$
\lambda_{t}(D)=\text { ess. } \inf _{\mathbb{Q} \in \mathcal{P}} \mathbf{E}_{\mathbb{Q}}\left[\sum_{t \leq k \leq N} D_{k} \mid \mathcal{F}_{t}\right],
$$

that is in terms of cumulative cash-flows:

$$
\mu_{t}(Y)=\operatorname{ess.} \inf _{\mathbb{Q} \in \mathcal{P}} \mathbf{E}_{\mathbb{Q}}\left[Y_{N}-Y_{t-1} \mid \mathcal{F}_{t}\right] .
$$

This is in contrast with our theory which, dealing with

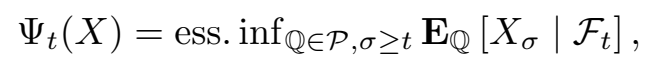

does not only look at final values but also looks at the whole trajectory.

\subsection{Time consistency of test probability sets (for value processes).}

Definition 4.1. A set $\mathcal{P}$ of test probabilities is "time consistent for value processes" if for each pair of value processes $X \in \mathcal{G}$ and $Y \in \mathcal{G}$ and each pair of stopping times $\sigma$ and $\tau, \sigma \leq \tau$, the two conditions

(i) $X^{\tau-}=Y^{\tau-}$,

(ii) $\Psi_{\tau}(X) \leq \Psi_{\tau}(Y)$, taken together, provide the inequality $\Psi_{\sigma}(X) \leq \Psi_{\sigma}(Y)$.

The notion goes back to Wang $(1996,1999)$, and we present it more generally, in terms of random (stopping) times and test probabilities.

The following theorem identifies stability with time consistency for value processes. It is related to decision theory with multipriors, see again Epstein and Schneider (2003). A corresponding version for risk measurement of final values, related in the finite $\Omega$ case to Riedel (2002) and Roorda, Engwerda and Schumacher (2002), will be established in Theorem 5.1.

Theorem 4.3. A set $\mathcal{P}$ of test probabilities is stable if and only if it is time consistent for value processes.

Proof. If $\mathcal{P}$ is time consistent we define for each $X \in \mathcal{G}$ and each stopping time $\tau$, the process $Y=X^{\tau-}+\Psi_{\tau}\left({ }^{\tau} X\right) \mathbf{1}_{\llbracket \tau, N \rrbracket}$ and we find that $X^{\tau-}=Y^{\tau-}, \Psi_{\tau}(X)=\Psi_{\tau}(Y)$. By time consistency we find for each stopping time $\sigma, \sigma \leq \tau$ that $\Psi_{\sigma}(X)=\Psi_{\sigma}(Y)$, which proves Bellman's principle.

For a stable $\mathcal{P}$, Bellman's principle together with conditions (i), (ii), and monotonicity of $\Psi_{\sigma}$ ensures that

$$
\begin{aligned}
\Psi_{\sigma}(X) & =\Psi_{\sigma}\left(X^{\tau-}+\Psi_{\tau}\left({ }^{\tau} X\right) \mathbf{1}_{\llbracket \tau, N \rrbracket}\right) \\
& =\Psi_{\sigma}\left(Y^{\tau-}+\Psi_{\tau}(X) \mathbf{1}_{\llbracket \tau, N \rrbracket}\right) \\
& \leq \Psi_{\sigma}\left(Y^{\tau-}+\Psi_{\tau}(Y) \mathbf{1}_{\llbracket \tau, N \rrbracket}\right) \\
& =\Psi_{\sigma}\left(Y^{\tau-}+\Psi_{\tau}\left({ }^{\tau} Y\right) \mathbf{1}_{\llbracket \tau, N \rrbracket}\right) \\
& =\Psi_{\sigma}(Y),
\end{aligned}
$$

which proves time consistency. 


\subsection{Relation with the construction in Section 2 .}

Proposition 4.1. For any set $\mathcal{P}$ of test probabilities, the deduced initial riskadjusted measurement $\Psi_{0}$ again generates $\Psi$ via the construction defined in Propositions 2.3, 2.4.

Proof. We indeed have for each value process $X$ and each stopping time $\rho$,

$$
\begin{aligned}
\Psi_{\rho}(X) & =\operatorname{ess.} \inf _{\mathbb{Q} \in \mathcal{P}, \tau \geq \rho} \mathbf{E}_{\mathbb{Q}}\left[X_{\tau} \mid \mathcal{F}_{\rho}\right] \\
& =\operatorname{ess} \sup \left\{g \in \mathcal{F}_{\rho} \mid \text { for all } \mathbb{Q} \in \mathcal{P} \text { and all } \tau \geq \rho: g \leq \mathbf{E}_{\mathbb{Q}}\left[X_{\tau} \mid \mathcal{F}_{\rho}\right]\right\} .
\end{aligned}
$$

The last condition on $g$ can also be written as: for each $A \in \mathcal{F}_{\rho}, \mathbb{Q} \in \mathcal{P}^{e}, \tau \geq$ $\rho, \mathbf{E}_{\mathbb{Q}}\left[\mathbf{1}_{A}\left(X-g \mathbf{1}_{\llbracket \rho, N \rrbracket}\right)_{\tau}\right] \geq 0$. This means that $\psi_{0}\left(\mathbf{1}_{A}\left(X-g \mathbf{1}_{[\rho, N \rrbracket}\right)\right) \geq 0$.

Remark. This proposition shows that it is possible to "go" from the $\mathcal{P}$ approach of risk measurement to the $\mathcal{A}$ approach of Propositions 2.1, 2.2 and 2.3. Work remains to be done concerning the reverse direction.

\section{MULTIPERIOD-MEASUREMENT OF FINAL VALUES}

This section deals with multiperiod risk-measurement of a "final value" (bounded $\mathcal{F}_{N}$-measurable random variable) for which a whole process of risk-adjusted values will be built. One may consult Wilkie, Waters and Yang (2003), Sections 3.4.2 and 3.4.3, for an example of early discussion (ca. 1980) of multiperiod "reserves", i.e. capital requirements, for such a "locked-in" position.

\subsection{Direct and derived constructions of a risk-adjusted value process.}

In earlier versions of this paper we gave in detail, out of a given set $\mathcal{P}$ of test probabilities, two constructions of processes of risk-adjusted values. Here we shall rather connect such constructions to the definitions of $\Psi$ and $\bar{\Psi}$ of Sections 4.1 and 4.2. For this we use the following

Definition 5.1. A bounded $\mathcal{F}_{N}$-random variable $f$ is called a "final value" and we denote by $X_{f}$ the element of $\mathcal{G}$ such that $\left(X_{f}\right)_{N}=f,\left(X_{f}\right)_{n}=\|f\|$ for $n<N$. For each stopping time $\sigma$ we define

$$
\Phi_{\sigma}(f)=\Psi_{\sigma}\left(X_{f}\right), \bar{\Phi}_{\sigma}(f)=\bar{\Psi}_{\sigma}\left(X_{f}\right) .
$$

Proposition 5.1. For each "final value" $f$ we have

$$
\begin{aligned}
& \Phi_{\tau}(f)=\text { ess. } \inf _{\mathbb{Q} \in \mathcal{P} e} \mathbf{E}_{\mathbb{Q}}\left[f \mid \mathcal{F}_{\tau}\right] \text { for each stopping time } \tau \\
& \bar{\Phi}_{n}(f)=\text { ess. } \inf _{\mathbb{Q} \in \mathcal{P}^{e}} \mathbf{E}_{\mathbb{Q}}\left[\bar{\Phi}_{n+1}(f) \mid \mathcal{F}_{n}\right], 0 \leq n<N, \bar{\Phi}_{N}(f)=f .
\end{aligned}
$$

We leave the proof, based on the following equalities, to the reader

$$
\begin{aligned}
& \text { ess. } \inf _{\mathbb{Q} \in \mathcal{P} e} \mathbf{E}_{\mathbb{Q}}\left[f \mid \mathcal{F}_{n}\right] \\
& =\text { ess. } \sup \left\{g \mid \text { for all } \mathbb{Q} \in \mathcal{P}: g \leq \mathbf{E}_{\mathbb{Q}}\left[f \mid \mathcal{F}_{n}\right], \mathbb{Q} \text { a.s. }\right\} \\
& =\text { ess. } \sup \left\{g \mid \text { for all } \mathbb{Q} \in \mathcal{P}^{e}: g \leq \mathbf{E}_{\mathbb{Q}}\left[f \mid \mathcal{F}_{n}\right], \mathbb{Q} \text { a.s. }\right\} .
\end{aligned}
$$




\subsection{Time consistency of test probability sets (for final values).}

We want to single out test probability sets for which "acceptability tomorrow in any event" ensures "acceptability today".

Definition 5.2. A set $\mathcal{P}$ of test probabilities is called "time consistent for final values" if for each pair of stopping times $\sigma \leq \tau$ and each pair of final values $f, g$, we have that $\Phi_{\tau}(f) \leq \Phi_{\tau}(g)$ implies that $\Phi_{\sigma}(f) \leq \Phi_{\sigma}(g)$.

Remark. Notice that without any assumption on $\mathcal{P}$ the inequality $\Phi_{\tau}(g) \geq 0$ implies that for $\sigma \leq \tau, \Phi_{\sigma}(g) \geq 0$, a weaker form of time consistency. It will be shown in Section 5.3 that the tail value-at-risk measurement does not satisfy this weak form of time consistency.

The equivalence between Definition 4.1 and Definition 5.2 will follow from the Theorem 5.1. The following theorem is clearly similar to Theorem 4.2 and identifies stability with time consistency.

Theorem 5.1. The properties (1) to(5) below are equivalent.

(1) stability of the set $\mathcal{P}$,

(2) recursivity: for each final value $f$, the family $\left\{\Phi_{\nu}(f) \mid \nu\right.$ a stopping time $\}$ satisfies: for every two stopping times $\sigma \leq \tau$ we have $\Phi_{\sigma}(f)=\Phi_{\sigma}\left(\Phi_{\tau}(f)\right)$,

(3) for each final value $f$, for every stopping time $\sigma$ we have $\Phi_{0}(f) \leq \Phi_{0}\left(\Phi_{\sigma}(f)\right)$,

(4) time consistency of the set $\mathcal{P}$ for final values,

(5) for each final value $f$ the family of the $\Phi_{\sigma}(f)$ satisfies the submartingale property: for each $\mathbb{Q} \in \mathcal{P}$ and each pair of stopping times $\sigma \leq \tau$ we have that $\Phi_{\sigma}(f) \leq \mathbf{E}_{\mathbb{Q}}\left[\Phi_{\tau}(f) \mid \mathcal{F}_{\sigma}\right]$.

Remark 1. Property (2) would be written in terms of risk measures as $\rho_{\sigma}(f)=$ $\rho_{\sigma}\left(-\rho_{\tau}(f)\right)$. With three periods we would get formulas like $\rho_{0}(f)=\rho_{0}\left(-\rho_{1}\left(-\rho_{2}(f)\right)\right)$ and, again, it is better to work with the "risk-adjusted value" $\pi=-\rho$.

Remark 2. The implication of (2) by (3) means that it suffuces to check the date 0 risk-adjusted value against the adjustment of any future adjusted value to get the stability and the time consistency properties.

Proof. Let us show that (4) follows from (2). Suppose that for two bounded random variables $f, g$ and two stopping times $\sigma \leq \tau$, we have $\Phi_{\tau}(f) \leq \Phi_{\tau}(g)$. Then we have by monotonicity of $\Phi_{\sigma}$, if (2) holds, $\Phi_{\sigma}(f)=\Phi_{\sigma}\left(\Phi_{\tau}(f)\right) \leq \Phi_{\sigma}\left(\Phi_{\tau}(g)\right)=\Phi_{\sigma}(g)$.

Conversely to prove (2) out of (4), we take for the two random variables, the functions $f$ and $g=\Phi_{\tau}(f)$. We have equality $\Phi_{\tau}\left(\Phi_{\tau}(f)\right)=\Phi_{\tau}(f)$ and therefore (applying item $(2)$ twice) that $\Phi_{\sigma}\left(\Phi_{\tau}(f)\right)=\Phi_{\sigma}(f)$.

Obviously (2) implies (3).

We show now that (3) implies (1); this is the same as in Theorem 4.2. We suppose that $Z^{1}$ and $Z^{2}$ are two elements in $\mathcal{P}^{e}$ - coming from the measures $\mathbb{Q}^{1}, \mathbb{Q}^{2}$ - and $\sigma$ is a stopping time. Also suppose that the element $Z_{\sigma}^{1} \frac{Z_{N}^{2}}{Z_{\sigma}^{2}}$ is not in the closed convex set $\mathcal{P}$. By the separation theorem for convex sets there is a random variable $f \in L^{\infty}$, so that

$$
\mathbf{E}_{\mathbb{P}}\left[Z_{\sigma}^{1} \frac{Z_{N}^{2}}{Z_{\sigma}^{2}} f\right]<\inf _{\mathbb{Q} \in \mathcal{P}} \mathbf{E}_{\mathbb{Q}}[f] .
$$

We can write the left hand side as $\mathbf{E}_{\mathbb{Q}^{1}}\left[\mathbf{E}_{\mathbb{Q}^{2}}\left[f \mid \mathcal{F}_{\sigma}\right]\right]$. This is clearly at least equal to $\mathbf{E}_{\mathbb{Q}^{1}}\left[\Phi_{\sigma}(f)\right]$, a quantity at least equal to $\Phi_{0}\left[\Phi_{\sigma}(f)\right]$, hence by property $(3)$, at 
least equal to $\Phi_{0}(f)$. This is a contradiction since the right hand side is precisely $\Phi_{0}(f)$.

If (2) holds we take $g=\Phi_{\tau}(f)$ in the definition of $\Phi_{\sigma}(g)$ to find that for each $\mathbb{Q} \in \mathcal{P}, \Phi_{\sigma}(f) \leq \mathbf{E}_{\mathbb{Q}}\left[\Phi_{\tau}(f) \mid \mathcal{F}_{\sigma}\right]$ which proves $(5)$.

Suppose now that the qualitative condition (5) holds: "risk-adjusted values increase in conditional mean over time" and let us prove (3). For each $f \in L^{\infty}$ and each pair $\sigma \leq \tau$ of stopping times we have $\Phi_{\sigma}(f) \leq \mathbf{E}_{\mathbb{Q}}\left[\Phi_{\tau}(f) \mid \mathcal{F}_{\sigma}\right]$, hence $\Phi_{\sigma}(f) \leq \Phi_{\sigma}\left(\Phi_{\tau}(f)\right)$. Since always $\Phi_{\sigma}(f) \geq \Phi_{\sigma}\left(\Phi_{\tau}(f)\right)$, we have equality.

Since by Theorem 4.2, (1) implies Bellman's principle, the proof of the theorem shall be completed by showing that Bellman's principle implies time consistency (2) for values. This results from the relation

$$
\begin{aligned}
\Phi_{\sigma}(f) & =\Psi_{\sigma}\left(X_{f}\right)=\Psi_{\sigma}\left(\left(X_{f}\right)^{\tau-}+\Psi_{\tau}\left({ }^{\tau}\left(X_{f}\right)\right) \mathbf{1}_{\llbracket \tau, N \rrbracket}\right) \\
& =\Psi_{\sigma}\left(\|f\|, \ldots,\|f\|, \Psi_{\tau}\left(X_{f}\right), \ldots, \Psi_{\tau}\left(X_{f}\right)\right) \\
& \left.=\Phi_{\sigma}\left(\Psi_{\tau}\left(X_{f}\right)\right)\right)=\Phi_{\sigma}\left(\Phi_{\tau}(f)\right) .
\end{aligned}
$$

Remark. We do not want to reduce the work of Section 4 to the one in Sections 5.1 and 5.2. The way cash-flows received or paid at date $n$ are transported to date $N$ is, in our opinion, part of the strategy to be risk-measured. We have provided tools to take into account what could happen along trajectories. And all of this is to be appreciated for example at date 0 .

\subsection{Discussing the Tail Value-at-Risk measurement.}

Tail Value-at-Risk, see Embrechts (1995), Artzner et al. (1997, 1999a), Delbaen (2000, 2002), has been well received, in particular in credit risk measurement and insurance supervision (see Wilkie, Waters and Yang (2003)), as it gives an idea of "how bad is bad". Here we explain why care must be used in the multiperiod setting, as we had warned in Artzner (2002a).

The good definition of Tail Value-at-Risk of level $\alpha$ for the one-period case reads as follows:

$$
T \operatorname{TV} a R^{\alpha}(X)=\inf _{\mathbb{Q}}\left\{\mathbf{E}_{\mathbb{Q}}[X] \mid \frac{d \mathbb{Q}}{d \mathbb{P}_{0}} \leq \alpha^{-1}\right\}
$$

that is also:

$$
T \operatorname{VaR} R^{\alpha}(X)=\inf \left\{\mathbf{E}_{\mathbb{P}_{0}}[Z X] \mid 0 \leq Z \leq \alpha^{-1}, \mathbf{E}_{\mathbb{P}_{0}}[Z]=1\right\} .
$$

Definition 5.5. Given a final value $f$ and dates $n, n+h, 0 \leq n \leq n+h \leq N$, Tail Value-at-Risk for the time interval $(n, n+h)$ at level $\alpha$ is given by $T V a R_{n, n+h}^{\alpha}(f)=$ ess. $\inf \left\{\mathbf{E}_{\mathbb{P}_{0}}\left[Z f \mid \mathcal{F}_{n}\right] \mid 0 \leq Z \leq \alpha^{-1}, \mathbf{E}_{\mathbb{P}_{0}}\left[Z \mid \mathcal{F}_{n}\right]=1, Z\right.$ is $\mathcal{F}_{n+h}$-measurable $\}$.

Remark. Notice that for $n+h=N$ fixed and $n$ variable, we use different sets of test probabilities.

We first notice that the set of test probabilities providing the coherent one-period risk-adjusted measurement $T V a R_{0, N}^{\alpha}$ is not stable, see Artzner (2002a). We also show that the system of the $\left(T V a R_{n, N}^{\alpha}\right)_{n}$ does not come from a mapping like $\Phi$ or $\bar{\Phi}$ of Section 5.1, since the weak form of time consistency (see the remark after Definition 5.2) is not even satisfied. Here is an example of a future value $g$ at date $N=2$ with a "TailVaR" positive as random variable at date 1 and negative at date 0 :

$\Omega=\{[u u],[u m],[u d],[d u],[d m],[d d]\}, \mathbb{P}_{0}$ uniform on $\Omega, \mathcal{F}_{1}$ generated by $[\mathrm{u}]$ and $[\mathrm{d}]$, 
$g([u u])=-10, g([u m])=12, g([u d]=14, g([d u])=-20, g([d m])=22, g([d d])=22$.

We find for $g$ the TVaR values, at the level $\alpha=\frac{2}{3}$, at date 0 and at date 1 :

$$
T \operatorname{Va} R_{1,2}^{\alpha}(g)([u])=T \operatorname{Va} R_{1,2}^{\alpha}(g)([d])=1, T \operatorname{Va} R_{0,2}^{\alpha}(g)=-1 .
$$

Remark. As shown in Delbaen(2001), risk adjusted values that are law invariant, such as Tail VaR, pose serious problems in a continuous time model. If a law invariant risk adjusted value is time consistent then - under quite general assumptions it equals the minimum wealth over all states of nature. 
* This research has benefited from support by the National Science Foundation under Grant No. 0139911, by RiskLab, ETH Zürich and by PriceWaterhouseCoopers. Preliminary presentations have been made at various academic and business meetings since February 2001: ETH Zürich; ORFE Dept., Princeton U.; Technische Universität Dresden; Carnegie Mellon U.; Swiss Re New Markets; Judge Institute of Management; Université Louis Pasteur; IIR Conference on Volatility and Risk, London; LSE Dept. of Statistics; Vienna University of Technology; Séminaire d'Actuariat, Lausanne; Crest-Insee, Paris; U. of Waterloo; Heriot Watt U.; Berg en Dal Meeting on functional analysis; Operations Research Kolloquium, Klagenfurt; MFS Meeting, Paphos; University of Cyprus; Université Paris-Dauphine; Fields Institute, Toronto; QMF Meeting, Cairns; MFO Tagung, Oberwolfach; Hitotsubashi U.; University of Besançon.

We thank the referees of earlier versions of this paper for useful remarks and suggestions. 


\section{REFERENCES}

Artzner, Philippe (2002a), Conditional Value at Risk: Is it good in the multiperiod case?, IIR Conference on Volatility and Risk, London, Feb. 18-19.

Artzner, Philippe (2002b), Multiperiod Risk Measurement: Where are we?, Quantitative Finance Seminar, Fields Institute, U. Toronto, Nov. 25, http://www.fields.utoronto.ca/audio/0203/finance_seminar/artzner/.

Artzner, Philippe, Freddy Delbaen, Jean-Marc Eber and David Heath (1997), Thinking coherently, Risk 10, 68-71.

Artzner, Philippe, Freddy Delbaen, Jean-Marc Eber and David Heath (1999a), Coherent Risk Measures, Mathematical Finance 9, 203-228.

Artzner, Philippe, Freddy Delbaen, Jean-Marc Eber and David Heath (1999b), Risk management and capital allocation with coherent Measures of Risk, http://symposium.wiwi.unikarlsruhe.de/8thpapers/artzner.ps.

Artzner, Philippe, Freddy Delbaen, Jean-Marc Eber and David Heath (2001), Coherent Measures of Multiperiod Risk, Presentation at the Workshop New Ideas in Risk Management, Carnegie Mellon University, Aug. 25-26.

Artzner, Philippe, Freddy Delbaen, and Pablo Koch-Medina (2005), Risk measures and efficient use of capital, Working Paper ETH, Zürich.

Bennet, Oliver (2001), Reinventing RAROC, Risk 14, 112-113.

Cheridito, Patrick, Freddy Delbaen and Michael Kupper (2002), Convex measures of Risk for bounded càdlàg processes, Stochastic Process. Appli. 112, 1-22.

Cvitanić, Jaksa and Ioannis Karatzas (1999), On dynamic measures of risk, Finance and Stochastics 3, 451-482.

Chow, Yuan Shih, Herbert Robbins and David Siegmund (1972), Great Expectations: The Theory of Optimal Stopping, Houghton Mifflin, Boston, reprinted by Dover, New York (1992).

Delbaen, Freddy (2000), Coherent Risk Measures on General Probability Spaces, Advances In Finance and Stochastics, Essays in Honour of Dieter Sondermann, (2002), Springer, New York.

Delbaen, Freddy (2001), The structure of $m$-stable sets and in particular of the set of risk neutral measures, Working Paper ETH, Zürich, to be published in Séminaire de Probabilités.

Delbaen, Freddy (2002), Coherent Risk Measures, Lectures given at the Cattedra Galileiana, March 2000, Scuola Normale Superiore, Pisa.

Dothan, Michael (1990), Prices in Financial Markets, Oxford University Press, New York.

Embrechts, Paul (1995), A survival Kit to Quantile Estimation, UBS Quant Workshop, Zürich.

Epstein, Larry and Martin Schneider (2003), Recursive multiple-priors, Journal of Economic Theory 113, 1-31, earlier versions June 2001, April 2002.

Föllmer, Hans and Alexander Schied (2002), Convex measures of risk and trading constraints, Finance and Stochastics 6, 429-447.

Föllmer, Hans and Alexander Schied (2004), Stochastic Finance, 2nd ed. de Gruyter, Berlin.

Gilboa, Itzhak and David Schmeidler (1989), Maxmin expected utility with non-unique prior, Jounal of Mathematical Economics 18, 141-153.

Jaschke, Stefan and Uwe Küchler (2001), Coherent risk measures and good-deal bounds, Finance and Stochastics 5, 181-200.

Heath, David (1998), Coherent Measures of Risk, Documents from the 5th Annual Conference on Risk Management, International Center for Business Information, Geneva, December 8th.

Nakano, Yumiharu (2003), Minimizing coherent risk measures of shortfall in discrete-time models with cone constraints, Applied Mathematical Finance 10, 163-181.

Neveu, Jacques (1972), Martingales à temps discret, Masson, Paris; English transl. DiscreteParameter Martingales, (1975), North-Holland.

Riedel, Frank (2002), Dynamic Coherent Risk Measures, Stochastic Processes and Applications 112, 185-200, earlier version November 2002.

Roorda, Berend, Jacob Engwerda and Hans Schumacher (2002), Coherent Acceptability Measures in Multiperiod Models, Working Paper U. of Twente and Tilburg U., June, later version July 2003.

Wang, Tan (1996), A Characterization of Dynamic Risk Measures, Working Paper Faculty of Commerce and Business Administration, U.B.C. 
Wang, Tan (1999), A Class of Dynamic Risk Measures, Working Paper Faculty of Commerce and Business Administration, U.B.C, later version 2002.

Wang, Tan (2003), Conditional preferences and Updating, Journal of Economic Theory 108, 286321, earlier version March 2002.

Wilkie, David, Howard Waters and Sheauwen Yang (2003), Reserving, Pricing and Hedging for Policies with Guaranteed Annuity Options, Paper presented to the Faculty of Actuaries, Edinburgh, 20 January 2003, British Actuarial Journal 9, 263-425. 